Research Paper

\title{
Comparison of the MAID (Al) and CAV/IE regimens with the predictive value of cyclic AMP-responsive element-binding protein 3 like protein 1 (CREB3L1) in palliative chemotherapy for advanced soft-tissue sarcoma patients
}

\author{
Wei Xiao ${ }^{1,3^{*}}$, Yao Liang $2,3^{*}$, Yi Que ${ }^{1,3^{*}}$, Jingjing Li ${ }^{1,3}$, Ruiqing Peng ${ }^{1,3}$, Bushu $\mathrm{Xu}^{1,3}$, Xizhi Wen ${ }^{1,3}$, Jingjing \\ Zhao $^{1,3}$, Yuanxiang Guan ${ }^{2,3}$, Xing Zhang ${ }^{1,3 凶}$ \\ 1. Melanoma and Sarcoma Medical Oncology Unit, Sun Yat-sen University Cancer Center, Guangzhou 510060, China. \\ 2. Department of Gastric Surgery, SunYat-sen University Cancer Center; State Key Laboratory of Oncology in South China; Collaborative Innovation Center \\ for Cancer Medicine. \\ 3. State Key Laboratory of Oncology in South China; Collaborative Innovation Center for Cancer Medicine, Sun Yat-sen University Cancer Center; Guangzhou \\ 510060, China. \\ ${ }^{*}$ Co-first authors.
}

$\triangle$ Corresponding author: Xing Zhang M.D \& PhD. Melanoma and Sarcoma Medical Oncology Unit, Sun Yat-sen University Cancer Center, Guangzhou 510060, China. Address: 651 Dongfeng East Road, Guangzhou 510060, P. R. China. E-mail: zhangxing@sysucc.org.cn; Tel.: 86-020-87343629

(C) Ivyspring International Publisher. This is an open access article distributed under the terms of the Creative Commons Attribution (CC BY-NC) license (https://creativecommons.org/licenses/by-nc/4.0/). See http://ivyspring.com/terms for full terms and conditions.

Received: 2018.07.24; Accepted: 2019.05.05; Published: 2019.06.09

\begin{abstract}
Background: Palliative chemotherapy is currently the first-line treatment for advanced soft tissue sarcoma. The purpose of this study was to compare the efficacies of the MAID (AI) and CAV/IE alternating regimens in advanced soft-tissue sarcoma patients. Since resistances to ADM-based chemotherapy and toxicity from doxorubicin are frequently observed in clinical practice, we investigated the association between CREB3L1 expression and survival in advanced soft-tissue sarcomas patients treated with doxorubicin-based palliative chemotherapy.

Methods: The cohort under investigation comprised 152 patients who underwent doxorubicin-based first-line palliative chemotherapy for advanced soft-tissue sarcoma at our institution between January 2010 and April 2017. Immunohistochemical analysis and the reverse transcription polymerase chain reaction were used to determine the expression of CREB3LI in soft-tissue sarcoma specimens prior to first-line palliative chemotherapy. Univariate and multivariate analyses were performed on chemotherapy regimens and CREB3LI expression levels. The relationship between CREB3LI expression and survival was also analyzed.

Results: The CAV/IE alternating regimen yielded favorable outcomes for response and survival in patients compared with those who received MAID (AI) treatment. The most common toxicity of grades 3 and 4 was leukopenia (58.5\% in the MAID (AI) regimen; $37.1 \%$ in the CAV/IE regimen). The incidence of febrile neutropenia after CAV/IE treatment (7.1\%) was lower than after MAID (AI) treatment (13.4\%). Grade 3 neuralgia was observed in $1.2 \%$ of patients receiving the MAID regimen versus $8.6 \%$ in patients receiving the CAV/IE regimen. High CREB3LI expression was observed in 48 of 152 patients (31.6\%). Overall survival was significantly higher for CREB3LI high-expression patients than for CREB3LI low-expression patients, especially for those also treated with the MAID (AI) regimen. The CREB3LI expression level was identified as an independent prognostic factor for survival by multivariate analysis.

Conclusions: Our study suggests that the CAV/IE alternating regimen may be associated with a better response and more favorable survival than the MAID (AI) regimen in advanced soft-tissue sarcoma patients. Furthermore, the CREB3L1 expression level may predict the efficacy and survival of doxorubicin-based palliative chemotherapy for advanced soft-tissue sarcoma.
\end{abstract}

Key words: Doxorubicin-based chemotherapy, CREB3L1, Soft-tissue sarcoma, Prognostic marker 


\section{Introduction}

Soft-tissue sarcomas are a heterogeneous group of relatively rare mesenchymal neoplasms.[1] Although typically incurable, patients with advanced soft-tissue sarcomas may derive some benefit from the appropriate use of chemotherapy.[2] Single-agent doxorubicin or doxorubicin-based combination regimens have been widely used to treat advanced soft-tissue sarcomas for decades.[3]

Therapeutic regimens predominantly containing doxorubicin are currently first-line chemotherapies used to treat advanced soft-tissue sarcomas.[4] The most commonly used palliative chemotherapeutic regimen is a combination of mesna, doxorubicin, ifosfamide, dacarbazine (MAID (AI)), which was compared with doxorubicin and DTIC in randomized trials for advanced sarcomas.[3] Arndt et al. explored an alternating regimen of cyclophosphamide, doxorubicin and vincristine (CAV), and ifosfamide and etoposide (IE) in intermediate-risk rhabdomyosarcoma and undifferentiated sarcoma.[5] The National Comprehensive Cancer Network $(\mathrm{NCCN})$ recommends this $\mathrm{CAV} / \mathrm{IE}$ alternating regimen as the first-line systemic therapy for non-pleomorphic rhabdomyosarcoma based on an updated report written by Arndt et al.[6] However, there are no studies directly comparing the MAID (AI) and CAV/IE alternating regimens.

Additionally, some patients with soft-tissue sarcomas do not benefit from doxorubicin-based palliative chemotherapy.[7, 8] Therefore, selecting patients with advanced soft-tissue sarcomas who are expected to show good responses to doxorubicin-based palliative chemotherapy and achieve favorable survival is important.[9-11] Various pathological factors have been reported as prognostic markers of poor survival in patients with soft-tissue sarcomas; however, these factors are inadequate for predicting survival in soft-tissue sarcomas patients. Therefore, molecular markers that predict survival and response in advanced soft-tissue sarcomas patients treated with doxorubicin-based palliative chemotherapy are needed.

Cyclic AMP-responsive element-binding protein 3 like protein 1 (CREB3L1) belongs to a family of transcription factors that are synthesized as membrane-bound precursors in the endoplasmic reticulum (ER).[12] CREB3L1 is then transported to the Golgi, where it becomes activated by regulated intramembrane proteolysis (RIP).[13] Using tumor cells cultured in vitro, Jing et al. reported that doxorubicin inhibited the proliferation of cancer cells expressing CREB3L1 but not that of those in which the gene was not expressed.[14] These findings led us to hypothesize that the presence of CREB3L1 may predict clinical responses to doxorubicin-based chemotherapy in advanced soft-tissue sarcomas. Therefore, the present study examined the association between CREB3L1 expression and the efficacy of doxorubicin-based palliative chemotherapy in clinical practice for patients with advanced soft-tissue sarcoma.

\section{Methods}

\section{Patients and samples}

The cohort under investigation comprised 152 patients who underwent doxorubicin-based first-line palliative chemotherapy for advanced soft-tissue sarcoma at our institution between January 2010 and April 2017. Archival paraffin-embedded tissue and clinical data from patients with complete follow-up were available. The diagnostic work-up included the initial pathological sectioning of soft-tissue sarcoma, magnetic resonance imaging (MRI) and computed tomography (CT). Tumors were graded histologically in accordance with World Health Organization (WHO) classifications per the FNCLCC grading system.

The investigated variables recorded included sex, age, histological type, FNCLCC histological grade, tumor size, primary site, lung metastases and chemotherapy regimen (Table 1). Eighty-three (54.6 $\%)$ patients were male, and eighty-four (55.3\%) patients were greater than 40 years old. Among the 152 patients, 83 (54.6\%) exhibited tumor sizes larger than $5 \mathrm{~cm}$, and 105 (69.1\%) had lung metastases.

Palliative chemotherapy was performed only after the patient consented to therapy, and written informed consent was obtained from all patients. Anticancer agents administered as first-line palliative chemotherapy consisted of doxorubicin at $50 \mathrm{mg} / \mathrm{m}^{2}$. The doxorubicin-based chemotherapy regimens included a combination treatment of mesna, doxorubicin, ifosfamide and dacarbazine (MAID (AI)) or an alternating regimen of cyclophosphamide, doxorubicin and vincristine (CAV), and ifosfamide and etoposide (IE). In the present study, 82 patients received the MAID (AI) regimen, and 70 patients received CAV/IE alternating chemotherapy. Responses to chemotherapy were evaluated using imaging results according to RECIST 1.1. The treatment toxicity was graded according to International Common Toxicity Criteria (version 2.0). Clinical assessments of safety, including a physical examination, performance status, blood chemistry, and urinalysis, were performed at baseline and before each cycle of treatment.

The primary endpoint of the present study was overall survival (OS); defined as the time from the administration of first-line palliative chemotherapy to 
the reported date of death or the most recent follow-up if the patient was alive. Progression-free survival (PFS) was the time from the administration of first-line palliative chemotherapy to the first progression or death. All patients were followed-up in the outpatient clinic or by telephone interview, and the mean follow-up time was 67.2 months. Ethical approval for this study was obtained from the Medical Ethics Committee of Sun Yat-sen University Cancer Center.

Table 1. Clinicopathological features of patients with advanced soft-tissue sarcoma.

\begin{tabular}{ll}
\hline & Patients $(\%)$ \\
\hline All patients & $152(100 \%)$ \\
Sex & \\
Male & $83(54.6 \%)$ \\
Female & $69(45.4 \%)$ \\
Age & \\
$<40$ years old & $84(55.3 \%)$ \\
>= 40 years old & $68(44.7 \%)$ \\
Histological type & \\
Rhabdomyosarcomas & $57(37.5 \%)$ \\
Synovial sarcomas & $33(21.7 \%)$ \\
Undifferentiated sarcomas & $30(19.7 \%)$ \\
Other STS entity & $32(21.1 \%)$ \\
FNCLCC histological grade & \\
Low grade (Score 1$)$ & $80(52.6 \%)$ \\
High grade (Score 2 \& 3) & $72(47.4 \%)$ \\
Tumor size & \\
$<5$ cm & $69(45.4 \%)$ \\
$\geq 5$ cm & $83(54.6 \%)$ \\
Primary site & \\
Head and neck & $58(38.2 \%)$ \\
Trunk and extremities & $94(61.8 \%)$ \\
Lung metastases & \\
No & \\
Yes & \\
Chemotherapy regimen & $70(30.9 \%)$ \\
MAID (AI) & \\
CAV / IE & \\
\hline & \\
\hline & \\
\hline
\end{tabular}

\section{Immunohistochemical analysis}

CREB3L1 expression levels were determined by immunohistochemical (IHC) staining of paraffinembedded tissue sections from pathological specimens prior to first-line palliative chemotherapy. Paraffin-embedded tissue sections were deparaffinized in xylene, and an alcohol gradient was utilized to rehydrate the sections. Next, sections were treated with a citrate antigen retrieval solution $(\mathrm{pH}=$ 6.0) in a pressure cooker for $5 \mathrm{~min}$. Subsequently, $5 \%$ bovine serum albumin (BSA) in PBS ( $25 \mathrm{mM}$ Tris, 0.8 $\% \mathrm{NaCl}, 2.68 \mathrm{mM} \mathrm{KCl}(\mathrm{pH} 7.4)$ ) was added to block non-specific binding, and sections were then incubated with a rabbit anti-CREB3L1 antibody (1:50, Proteintech, 11235-2-AP) in a moist chamber overnight at $4^{\circ} \mathrm{C}$. Secondary antibodies were incubated for $45 \mathrm{~min}$ at $37^{\circ} \mathrm{C}$ the next day. Finally, the sections were incubated in 3,3-diaminobenzidine for 2 min and counterstained with $10 \%$ Mayer's hematoxylin before being dehydrated and mounted. As a negative control, the primary antibody was replaced with phosphate buffered saline.

Stained tumor tissues were blindly evaluated with respect to clinical patient data and independently scored by two pathologists. Cytoplasmic staining was considered positive, and staining intensity was scored as 0,1 , or 2, corresponding to no, weak, and strong intensity, respectively (Figure 1). The percentage scores of cells showing cytoplasmic staining were also counted as follows: $0(<5 \%), 1(5-25 \%), 2(25-50 \%)$, or 3 (> 50 $\%)$. The total histochemical score (H-score $0-6)$ was calculated by multiplying the intensity score by the percentage score. Tumor tissues with $\mathrm{H}$-scores higher than 3 were considered to have high CREB3L1 expression. Tumors were classified as CREB3L1 low expression (H-score 0 - 3) or CREB3L1 high expression (H-score 4 - 6).
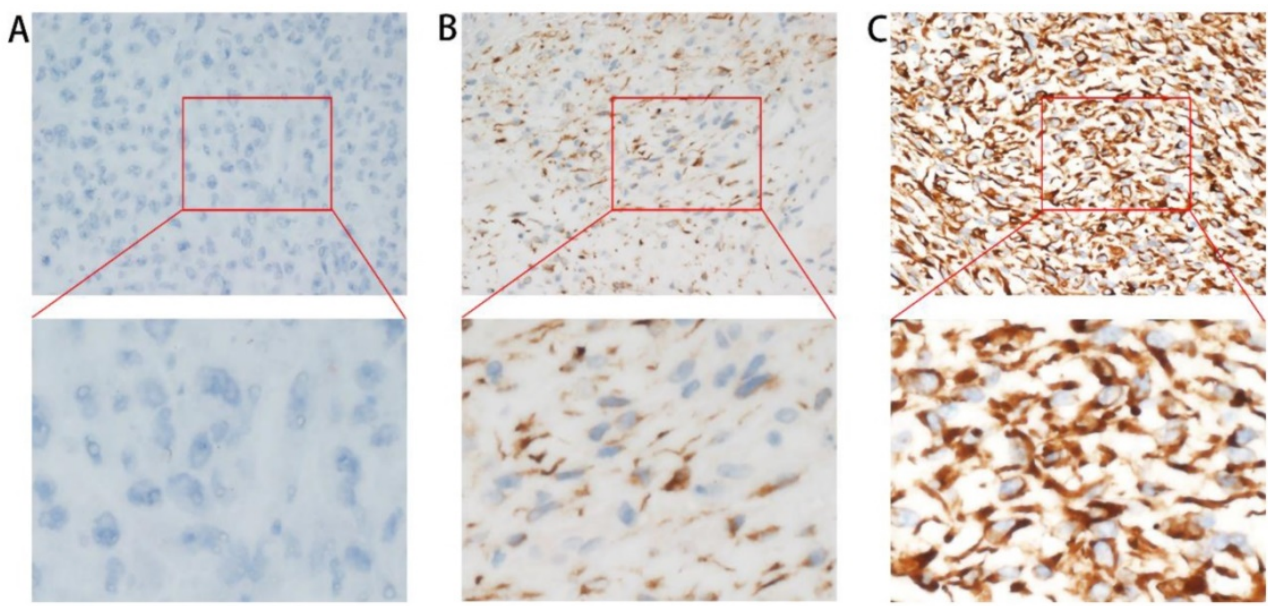

Figure 1. Immunohistochemical staining of paraffin-embedded sections before doxorubicin-based first-line palliative chemotherapy with an anti-CREB3L1 antibody. CREB3L1 protein expression was localized within the cytoplasm. Magnification 400x. A. CREB3L1-negative staining pattern (no intensity: score 0). B. CREB3L1-positive staining pattern (weak intensities: score 1). C. CREB3L1-positive staining pattern (strong intensities: score 2). 


\section{Real-time quantitative reverse transcription polymerase chain reaction ( $q R T-P C R$ )}

Total RNA was extracted from formalin-fixed paraffin-embedded tissue specimens using TRIzol reagent (Invitrogen, Carlsbad, CA, USA) according to the manufacturer's instructions. The reverse transcription kit (Promega, Madison, Wisconsin, USA) was used to synthesize complementary DNA (cDNA) from $2 \mu \mathrm{g}$ of total RNA. qRT-PCR was performed using Power SYBR Green qPCR SuperMix-UDG (Invitrogen, Carlsbad, CA, USA) to detect the mRNA levels of target genes with a LightCycler 480 II (Roche, Basel, Switzerland). $\beta$-actin was used as the internal control. All reactions were performed in triplicate over three independent experiments. The sequences of real-time PCR primers are as follows: CREB3L1 sense: ATGGACGCCG TCTTGGAACC; CREB3L1 anti-sense: CTAGGAGA GTTTGATGGTGG; $\beta$-actin sense: CGC GAG AAG ATG ACC CAG AT; and $\beta$-actin anti-sense: GGG CAT ACC CCT CGT AGA TG.

Table 2. Responses in patients with advanced soft-tissue sarcoma after doxorubicin-based first-line palliative chemotherapy.

\begin{tabular}{|c|c|c|c|}
\hline & \multicolumn{2}{|c|}{ Overall response } & \multirow[b]{2}{*}{$P$ value } \\
\hline & No & Yes & \\
\hline All patients & $78(51.3 \%)$ & $74(48.7 \%)$ & \\
\hline Sex & & & NS \\
\hline Male & $40(48.2 \%)$ & $43(51.8 \%)$ & \\
\hline Female & $38(55.1 \%)$ & $31(44.9 \%)$ & \\
\hline Age & & & 0.001 \\
\hline$<40$ years old & $33(39.3 \%)$ & $51(60.7 \%)$ & \\
\hline$\geq 40$ years old & $45(66.2 \%)$ & $23(33.8 \%)$ & \\
\hline Histological type & & & NS \\
\hline Rhabdomyosarcomas & $23(40.4 \%)$ & $34(59.6 \%)$ & \\
\hline Synovial sarcomas & $20(60.6 \%)$ & $13(39.4 \%)$ & \\
\hline Undifferentiated sarcomas & $18(60.0 \%)$ & $12(40.0 \%)$ & \\
\hline Other STS entity & $17(53.1 \%)$ & $15(46.9 \%)$ & \\
\hline FNCLCC grade & & & 0.022 \\
\hline Low grade (Score 1) & $34(42.5 \%)$ & $46(57.5 \%)$ & \\
\hline High grade (Score $2 \& 3$ ) & $44(61.1 \%)$ & $28(38.9 \%)$ & \\
\hline Tumor size & & & $<0.001$ \\
\hline$<5 \mathrm{~cm}$ & $24(34.8 \%)$ & $45(65.2 \%)$ & \\
\hline$\geq 5 \mathrm{~cm}$ & $54(65.1 \%)$ & $29(34.9 \%)$ & \\
\hline Primary site & & & NS \\
\hline Head and neck & $24(41.4 \%)$ & $34(58.6 \%)$ & \\
\hline Trunk and extremities & $54(57.4 \%)$ & $40(42.6 \%)$ & \\
\hline Lung metastases & & & NS \\
\hline No & $24(51.1 \%)$ & $23(48.9 \%)$ & \\
\hline Yes & $54(51.4 \%)$ & $51(48.6 \%)$ & \\
\hline Chemotherapy regimen & & & $<0.001$ \\
\hline MAID (AI) & $53(64.6 \%)$ & $29(35.4 \%)$ & \\
\hline $\mathrm{CAV} / \mathrm{IE}$ & $25(35.7 \%)$ & $45(64.3 \%)$ & \\
\hline CREB3L1 expression & & & 0.012 \\
\hline Low & $61(58.1 \%)$ & $44(41.9 \%)$ & \\
\hline High & $17(36.2 \%)$ & $30(63.8 \%)$ & \\
\hline
\end{tabular}

\section{Statistical analysis}

Associations between CREB3L1 expression and clinicopathological factors were analyzed using Fisher's exact test. Response rate, progression-free survival and overall survival were compared using the Chi-square test and the Kaplan-Meier method. Differences in survival among groups were compared using log-rank tests. We used a logistical model and Cox proportional hazards regression analysis to assess chemotherapeutic regimens and CREB3L1 expression in relation to response and survival. $P$-values $<0.05$ were considered statistically significant. All statistical analyses were performed using SPSS version 17.0 statistical software (IBM Corp, Armonk, NY, USA).

\section{Results}

\section{Comparison of the efficacies of the MAID (AI) and $C A V / I E$ regimens}

In this study, the overall response rate to doxorubicin-based first-line palliative chemotherapy in patients with advanced soft-tissue sarcoma was $48.7 \%$. The response rate differed significantly between the two regimens in favor of the CAV / IE regimen. Twenty-nine of eighty-two (35.4\%) patients in the MAID (AI) regimen group and forty-five of seventy $(64.3 \%)$ patients in the CAV / IE regimen group exhibited an overall response (Table 2; $P<0.001)$.

\section{Prognostic analysis of the MAID (AI) and CAVIIE regimens on survival}

Patients who received the $\mathrm{CAV} / \mathrm{IE}$ alternating regimen experienced a more favorable outcome with respect to progression-free survival in comparison with those who underwent MAID (AI) treatment (Figure 3A; $P=0.001$ ). The median progression-free survival was 6 months in the MAID (AI) regimen group versus 10 months in the CAV/IE alternative regimen group. Compared to patients who received the MAID (AI) regimen, there was increased overall survival in patients who received CAV/IE alternating regimens (Figure 3B; $P<0.001$ ). The overall survival at 1 year was $52.2 \%$ in the MAID (AI) regimen group versus $85.1 \%$ in the $\mathrm{CAV} / \mathrm{IE}$ alternative regimen group. The overall survival at 2 years was $13.1 \%$ in the MAID (AI) regimen group versus $57.5 \%$ in the $\mathrm{CAV} / \mathrm{IE}$ alternating regimen group. In multivariate analysis with possible confounding factors, chemotherapeutic regimen remained independent prognostic factors for both progression-free survival (Table 3; $P<0.001$ ) and overall survival (Table 3; $P=$ $0.001)$.

For patients with primary lesions in the head and neck, the CAV/IE regimen conveyed a more favorable overall survival compared with the MAID (AI) regimen $(P=0.036$; Table 4$)$. The overall survival in synovial sarcoma was significantly longer in the 
$\mathrm{CAV} / \mathrm{IE}$ alternative regimen group than in the MAID (AI) regimen group $(P=0.009$; Table 4$)$.

Table 3. Survival in patients with advanced soft-tissue sarcoma after doxorubicin-based first-line palliative chemotherapy.

\begin{tabular}{|c|c|c|c|c|}
\hline & \multicolumn{2}{|c|}{ Progression-free survival } & \multicolumn{2}{|l|}{ Overall survival } \\
\hline & HR $(95 \% \mathrm{CI})$ & $P$ value & $\mathrm{HR}(95 \% \mathrm{CI})$ & $P$ value \\
\hline \multicolumn{5}{|l|}{ All patients } \\
\hline Sex & & NS & & NS \\
\hline Male & NA & & NA & \\
\hline Female & NA & & NA & \\
\hline Age & & $<0.001$ & & 0.013 \\
\hline$<40$ years old & 1.00 (Reference) & & 1.00 (Reference) & \\
\hline$\geq 40$ years old & $2.25(1.47-3.46)$ & & $2.02(1.16-3.54)$ & \\
\hline Histological type & & 0.003 & & $<0.001$ \\
\hline Rhabdomyosarcomas & 1.00 (Reference) & & 1.00 (Reference) & \\
\hline Synovial sarcomas & $1.69(1.02-2.82)$ & & $2.53(1.36-4.71)$ & \\
\hline Undifferentiated sarcomas & $0.64(0.35-1.12)$ & & $0.55(0.25-1.17)$ & \\
\hline Other STS entity & $0.56(0.32-0.98)$ & & $0.87(0.43-1.78)$ & \\
\hline FNCLCC grade & & NS & & $<0.001$ \\
\hline Low grade (Score 1 ) & NA & & 1.00 (Reference) & \\
\hline High grade (Score 2 \& 3) & NA & & $2.36(1.47-3.79)$ & \\
\hline Tumor size & & NS & & 0.019 \\
\hline$<5 \mathrm{~cm}$ & NA & & 1.00 (Reference) & \\
\hline$\geq 5 \mathrm{~cm}$ & NA & & $1.87(1.11-3.17)$ & \\
\hline Primary site & & 0.006 & & $<0.001$ \\
\hline Head and neck & 1.00 (Reference) & & 1.00 (Reference) & \\
\hline Trunk and extremities & $1.91(1.21-3.02)$ & & $4.67(2.45-8.90)$ & \\
\hline Lung metastases & & 0.020 & & 0.003 \\
\hline No & 1.00 (Reference) & & 1.00 (Reference) & \\
\hline Yes & $1.79(1.10-2.91)$ & & $2.39(1.34-4.29)$ & \\
\hline Chemotherapy regimen & & 0.034 & & $<0.001$ \\
\hline MAID (AI) & 1.00 (Reference) & & 1.00 (Reference) & \\
\hline $\mathrm{CAV} / \mathrm{IE}$ & $0.62(0.40-0.97)$ & & $0.29(0.16-0.52)$ & \\
\hline CREB3L1 expression & & $<0.001$ & & 0.001 \\
\hline Low & 1.00 (Reference) & & 1.00 (Reference) & \\
\hline High & $0.43(0.27-0.67)$ & & $0.40(0.23-0.69)$ & \\
\hline
\end{tabular}

NS: not significant. NA: data not available.

Table 4. Overall survival in patients with head and neck primary lesions or synovial sarcomas after doxorubicin-based first-line palliative chemotherapy.

\begin{tabular}{lllll}
\hline & \multicolumn{2}{l}{ Head and neck primary lesion } & \multicolumn{2}{l}{ Synovial sarcoma } \\
\hline & $\begin{array}{l}\text { Median } \\
\text { OS }\end{array}$ & $\begin{array}{l}\text { P value for survival } \\
\text { curve }\end{array}$ & $\begin{array}{l}\text { Median } \\
\text { OS }\end{array}$ & $\begin{array}{l}\text { P value for survival } \\
\text { curve }\end{array}$ \\
\hline $\begin{array}{l}\text { MAID } \\
\text { regimen }\end{array}$ & $\begin{array}{l}\text { months } \\
\text { CAV } / \mathrm{IE}\end{array}$ & 0.036 & 9 months & 0.009 \\
regimen & $\begin{array}{l}12.5 \\
\text { months }\end{array}$ & 17 & \\
\hline
\end{tabular}

\section{Adverse events for the MAID (AI) and CAVIIE regimens}

The common toxicity (grade $\geq 3$ ) for first-line palliative chemotherapy in all 152 advanced soft-tissue sarcoma patients is summarized in Table 5 . Grade $\geq 3$ adverse events were more common in patients treated with the MAID (AI) regimen than in those treated with the $\mathrm{CAV} / \mathrm{IE}$ alternative regimen. The most common grade $\geq 3$ toxicity was leukopenia (58.5\% of patients with MAID (AI) regimen; $37.1 \%$ of patients with CAV/IE regimen). Febrile neutropenia was observed in 11 of 82 patients (13.4\%) from the MAID (AI) regimen and in 5 of 70 patients $(7.1 \%)$ from the $\mathrm{CAV} / \mathrm{IE}$ regimen. Grade 3 neuralgia was observed in $1.2 \%$ of patients receiving the MAID (AI) regimen versus $8.6 \%$ of patients receiving the $\mathrm{CAV} / \mathrm{IE}$ regimen.

Table 5. Common adverse events (grade $\geq 3$ ) after doxorubicin-based first-line palliative chemotherapy for advanced soft-tissue sarcoma.

\begin{tabular}{llll}
\hline Adverse event & Total & $\begin{array}{l}\text { MAID (AI) regimen } \\
=82)\end{array}$ & $\begin{array}{l}\text { CAV/IE regimen } \\
=70)\end{array}$ \\
\hline Leukopenia & $74(48.7 \%)$ & $48(58.5 \%)$ & $26(37.1 \%)$ \\
Febrile neutropenia & $16(10.5 \%)$ & $11(13.4 \%)$ & $5(7.1 \%)$ \\
Anemia & $6(3.9 \%)$ & $3(3.7 \%)$ & $3(4.3 \%)$ \\
Thrombocytopenia & $3(2.0 \%)$ & $1(1.2 \%)$ & $2(2.9 \%)$ \\
Neuralgia & $7(4.6 \%)$ & $1(1.2 \%)$ & $6(8.6 \%)$ \\
Nausea & $18(11.8 \%)$ & $8(9.8 \%)$ & $10(14.3 \%)$ \\
Mucositis & $7(4.6 \%)$ & $2(2.4 \%)$ & $5(7.1 \%)$ \\
Fatigue & $17(11.2 \%)$ & $9(11.0 \%)$ & $8(11.4 \%)$ \\
Diarrhea & $1(0.7 \%)$ & $1(1.2 \%)$ & $0(0.0 \%)$ \\
Vomiting & $10(6.6 \%)$ & $3(3.7 \%)$ & $7(10.0 \%)$ \\
\hline
\end{tabular}

\section{Assessment of CREB3L1 expression in advanced soft-tissue sarcoma}

CREB3L1 was localized to the cytoplasm in advanced soft-tissue sarcoma cells. Representative cases of different immunohistochemical staining levels are presented in Figure 1. Stained tumors with H-scores $\geq 3$ were deemed CREB3L1 high expression. Low expression of CREB3L1 was observed in one hundred and four specimens (68.4\%), whereas forty-eight CREB3L1 high-expression specimens (31.6 $\%$ ) were detected among the 152 advanced soft-tissue sarcoma patients.

CREB3L1 mRNA expression was assessed in 78 samples. We detected the levels of CREB3L1 mRNA in CREB3L1 high-expression and CREB3L1 low-expression samples as classified by IHC analysis. The relative mRNA levels of CREB3L1 were significantly higher in specimens with high CREB3L1 protein expression (mRNA $4.87 \pm 0.05$; Figure 2).

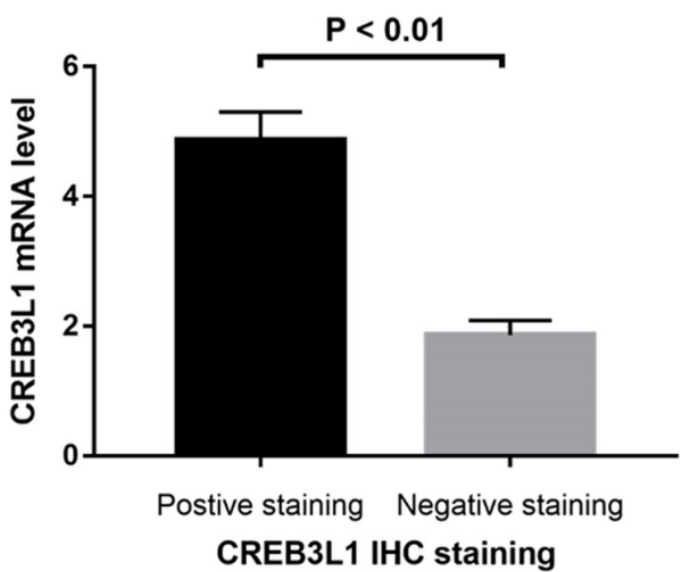

Figure 2. Relative levels of CREB3L1 mRNA in CREB3L1 high-expression and CREB3L1 low-expression cases as determined by IHC analysis. The CREB3L1 mRNA levels were significantly higher in CREB3L1 high-expression patients than in CREB3L1 low-expression patients $(P<0.01)$. 
A

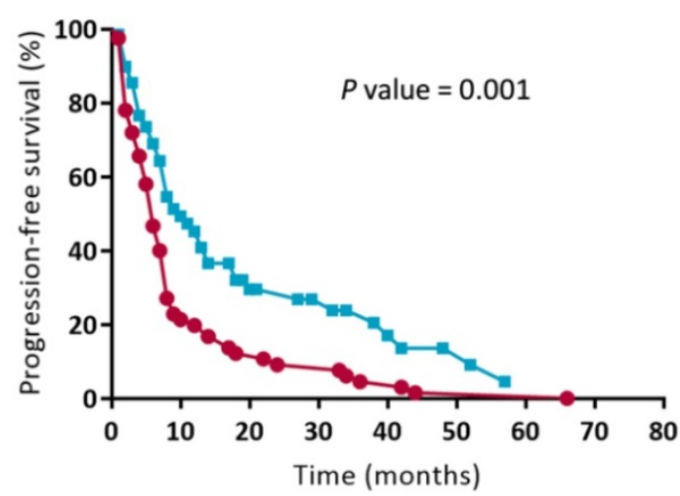

B

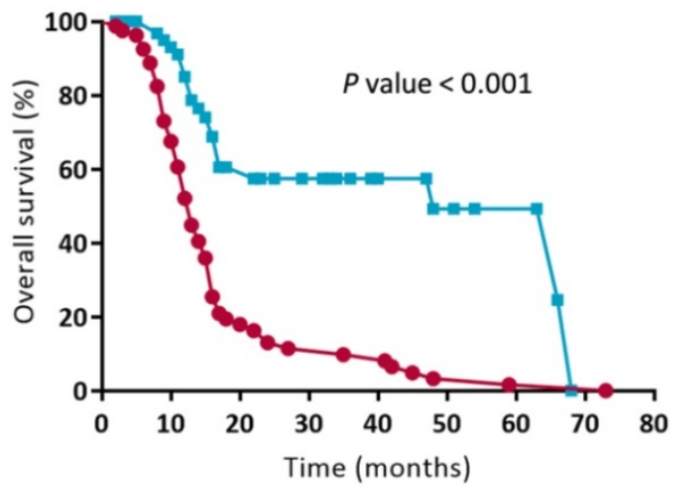

Figure 3. Kaplan-Meier survival analysis of patients being treated with the MAID (AI) or CAV/IE regimens. Differences in survival between the subgroups were analyzed by log-rank tests. Progression-free survival (A) and overall survival (B) were significantly longer for the CAV/IE alternating regimen than for the MAID (AI) regimen.

\section{Association between CREB3LI expression and patient characteristics}

The relationships between CREB3L1 expression and clinicopathological factors are summarized in Table 6. CREB3L1 expression was significantly associated with histological type $(P=0.016)$, FNCLCC histological grade $(P=0.028)$, and lung metastases $(P$ $=0.038)$. With respect to the histological type of advanced soft-tissue sarcoma, CREB3L1 high-expression specimens were found in $17.5 \%, 45.5$ $\%$ and $43.3 \%$ patients with rhabdomyosarcomas, synovial sarcomas and undifferentiated sarcomas, respectively. There were no significant associations observed between CREB3L1 expression and sex, age, tumor size, or primary site.

Table 6. CREB3Ll expression in patients with advanced soft-tissue sarcoma.

\begin{tabular}{|c|c|c|c|}
\hline & \multicolumn{2}{|c|}{ CREB3L1 expression } & \multirow[b]{2}{*}{ P value } \\
\hline & Low & High & \\
\hline All patients & $105(69.1 \%)$ & $47(30.9 \%)$ & \\
\hline Sex & & & NS \\
\hline Male & $57(68.7 \%)$ & $26(31.3 \%)$ & \\
\hline Female & $48(69.6 \%)$ & $21(30.4 \%)$ & \\
\hline Age & & & NS \\
\hline$<40$ years old & $55(65.5 \%)$ & $29(34.5 \%)$ & \\
\hline$\geq 40$ years old & $50(73.5 \%)$ & $18(26.5 \%)$ & \\
\hline Histological type & & & 0.016 \\
\hline Rhabdomyosarcomas & $47(82.5 \%)$ & $10(17.5 \%)$ & \\
\hline Synovial sarcomas & $18(54.5 \%)$ & $15(45.5 \%)$ & \\
\hline Undifferentiated sarcomas & $17(56.7 \%)$ & $13(43.3 \%)$ & \\
\hline Other STS entity & $23(71.9 \%)$ & $9(28.1 \%)$ & \\
\hline FNCLCC grade & & & 0.028 \\
\hline Low grade (Score 1 ) & $49(61.3 \%)$ & $31(38.8 \%)$ & \\
\hline High grade (Score 2 \& 3) & $56(77.8 \%)$ & $16(22.2 \%)$ & \\
\hline Tumor size & & & NS \\
\hline$<5 \mathrm{~cm}$ & $44(63.8 \%)$ & $25(36.2 \%)$ & \\
\hline$\geq 5 \mathrm{~cm}$ & $61(73.5 \%)$ & $22(26.5 \%)$ & \\
\hline Primary site & & & NS \\
\hline Head and neck & $44(75.9 \%)$ & $14(24.1 \%)$ & \\
\hline Trunk and extremities & $61(64.9 \%)$ & $33(35.1 \%)$ & \\
\hline Lung metastases & & & 0.038 \\
\hline No & $27(57.4 \%)$ & $20(42.6 \%)$ & \\
\hline Yes & $78(74.3 \%)$ & $27(25.7 \%)$ & \\
\hline
\end{tabular}

\section{Predictive analysis of CREB3LI expression for chemotherapeutic efficacy}

Thirty of forty-seven $(63.8 \%)$ patients with high CREB3L1 expression and forty-four of one hundred and five $(41.9 \%)$ patients with low CREB3L1 expression exhibited an overall response $(P=0.012$; Table 2). For the MAID (AI) regimen, the response rate of patients with high CREB3L1 expression (57.7 $\%$; 15 of 26) was greater than that for low CREB3L1 expression $(25.0 \% ; 14$ of 56$)$ (Table $7 ; P=0.004)$. For the $\mathrm{CAV} / \mathrm{IE}$ alternating regimen, although there was no significant association observed between the CREB3L1 expression and response rate $(P=0.414), 15$ of $21(71.4 \%)$ CREB3L1 high-expression patients exhibited overall responses compared with 30 of 49 (61.2 \%) CREB3L1 low-expression patients.

\section{Prognostic analysis of CREB3LI expression association with overall survival}

Overall survival was significantly longer for CREB3L1 high-expression patients than for CREB3L1 low-expression patients (Figure 4A; $P<0.001$ ). The overall survival at 1 year was $57.6 \%$ in the low CREB3L1 expression group versus $83.5 \%$ in the high CREB3L1 expression group. The median overall survival was 13 months in the low CREB3L1 expression group versus 24 months in the high CREB3L1 expression group. CREB3L1 expression remained an independent prognostic factor after multivariate analysis (Table 3). The overall survival for CREB3L1 high-expression patients was significantly longer than that for CREB3L1 low-expression patients in both the MAID (AI) regimen (Figure $4 \mathrm{~B} ; P=0.002$ ) and the $\mathrm{CAV} / \mathrm{IE}$ alternating regimen subgroups (Figure $4 C ; P=0.008$ ). The expression of CREB3L1 exhibited good prognostic significance for overall survival in patients with advanced soft-tissue sarcoma, particularly for those treated with the MAID (AI) regimen. 


\section{A}

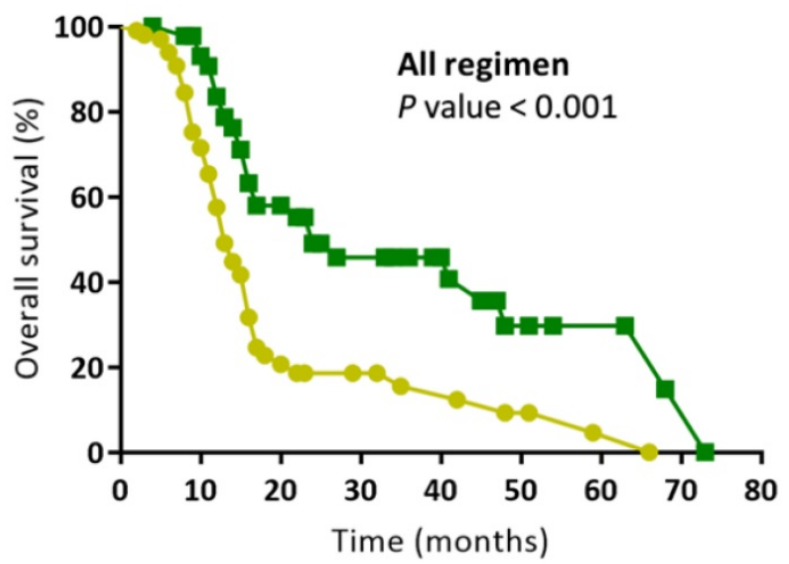

B
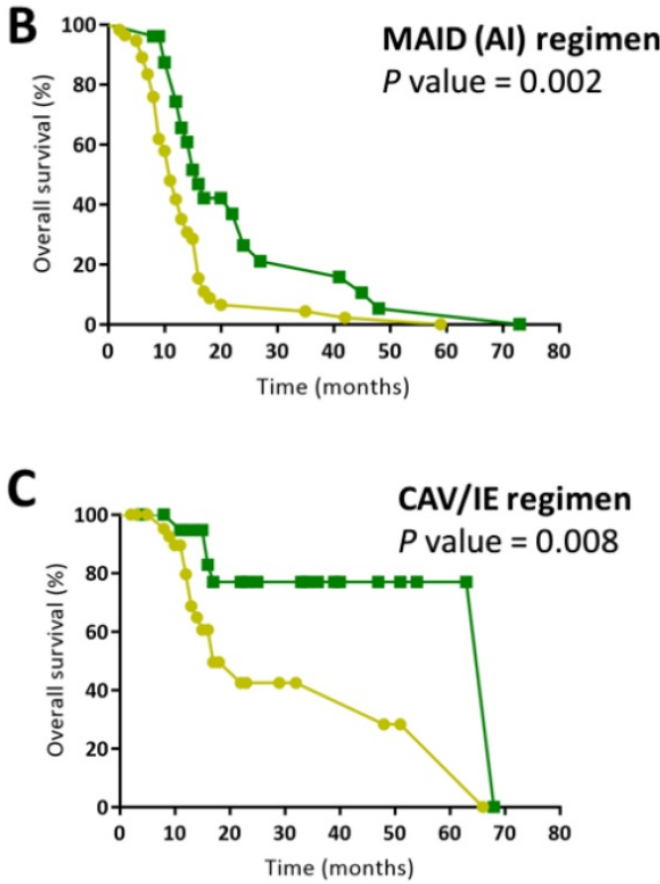

Figure 4. Kaplan-Meier survival analysis of patients with high or low levels of CREB3L1 expression. (A) Differences in overall survival between the subgroups were analyzed by log-rank tests. Overall survival was significantly longer for CREB3L1 high-expression patients than for CREB3L1 low-expression patients $(P<0.001)$. Kaplan-Meier survival analysis of patients who received the MAID(AI) regimen (B) or the CAV/IE alternative regimen (C).

Table 7. Responses to first-line palliative chemotherapy between CREB3L1 high-expression patients and CREB3L1 low-expression patients.

\begin{tabular}{|c|c|c|c|c|c|c|c|c|}
\hline \multirow[b]{2}{*}{ CREB3L1 expression } & \multicolumn{3}{|c|}{ MAID (AI) regimen } & \multirow[t]{2}{*}{$P$ value } & \multicolumn{3}{|c|}{$\mathrm{CAV} / \mathrm{IE}$ regimen } & \multirow[t]{2}{*}{ P value } \\
\hline & Total & Non-response & Response & & Total & Non-response & Response & \\
\hline Low & 56 & $42(75.0 \%)$ & $14(25.0 \%)$ & 0.004 & 49 & $19(38.8 \%)$ & $30(61.2 \%)$ & 0.414 \\
\hline High & 26 & $11(42.3 \%)$ & $15(57.7 \%)$ & & 21 & $6(28.6 \%)$ & $15(71.4 \%)$ & \\
\hline
\end{tabular}

\section{Discussion}

Doxorubicin and ifosfamide are currently the main therapeutic regimens used to treat advanced soft-tissue sarcomas $[15,16]$. The most common palliative chemotherapeutic regimen is the MAID (AI) regimen [3, 17], while the $\mathrm{CAV} / \mathrm{IE}$ alternating regimen is the first-line systemic therapy for rhabdomyosarcomas and PNET/Ewing's tumors [5, 6]. Because the CAV/IE alternating regimen may possess broad-spectrum antitumor activity with more chemotherapeutic agents than the MAID (AI) regimen, one of the purposes of the present study was to compare efficacies of the MAID (AI) and CAV/IE alternating regimens in advanced soft-tissue sarcomas patients.

The CAV/IE alternating regimen is indispensable for treating patients with advanced PNET/Ewing's sarcoma, rhabdomyosarcoma, undifferentiated sarcoma, or sarcoma of the bone $[5$, 18]. In addition, chemotherapy with cyclophosphamide, doxorubicin, vincristine, ifosfamide and etoposide is also a commonly used approach to small cell lung cancer and diffuse large B cell lymphoma $[19,20]$. Toxicity in the CAV/IE alternating regimen is quite tolerable [5], consistent with the results in this study. Even reducing the interval between chemotherapy cycles from three weeks to two weeks increased the favorable efficacy with no increase in toxicity [21]. The improvement in the outcome of patients receiving the CAV/IE alternating regimen may be due to the inhibition of topoisomerase II and, consequently, the impairment of DNA uncoiling after alkylating agent-induced DNA damage in response to ifosfamide [22].

There are no studies directly comparing the effects and toxicities of the MAID (AI) and CAV/IE alternating regimens in advanced soft-tissue sarcoma. In our study, the response rates differed significantly between the two groups in favor of the CAV/IE regimen. Patients exhibited a more favorable outcome with respect to both progression-free survival and overall survival in the $\mathrm{CAV} / \mathrm{IE}$ regimen in comparison to the MAID (AI) treatment. For patients with synovial sarcoma or rhabdomyosarcomas as well as patients with primary lesions in the head and neck, the $\mathrm{CAV} / \mathrm{IE}$ regimen also resulted in a more favorable overall survival in comparison with the MAID (AI) 
regimen. Since there is no effective chemotherapeutic regimen for patients with primary sarcoma lesions in the head and neck, the CAV/IE alternating regimen may be worth attempting. In addition, grade $\geq 3$ adverse events were less common in patients treated with the $\mathrm{CAV} / \mathrm{IE}$ alternating regimen than in those treated with the MAID (AI) regimen.

Concerning the resistance to ADM-based chemotherapy in clinical practice [23, 24], we investigated the associations between CREB3L1 expression and survival in advanced soft-tissue sarcomas patients treated with doxorubicin-based palliative chemotherapy. The present study revealed a significant association between CREB3L1 expression and the efficacy of doxorubicin-based first-line palliative chemotherapy for advanced soft-tissue sarcoma patients. The CREB3L1 expression, chemotherapy regimen and clinicopathological factors were included in the Cox proportional hazards model. High levels of CREB3L1 expression were associated with increased overall survival times in advanced soft-tissue sarcoma patients treated with doxorubicin-based chemotherapy in both the MAID (AI) regimen and the $\mathrm{CAV} / \mathrm{IE}$ alternative regimen. Using multivariate analysis, CREB3L1 expression levels emerged as an independent prognostic marker of survival. CREB3L1 may therefore predict prognosis independent of common prognostic factors, such as clinical $\mathrm{T}$ stage and histological grade.

CREB3L1 is an important factor in tumor development and metastasis as a transducer of the unfolded protein response. Transfecting CREB3L1 into cells that normally express low levels of CREB3L1 reduced cell migration, invasion, anchorageindependent growth and tolerance to hypoxia.[25] Deborah et al. reported the role of CREB3L1 as a metastasis suppressor in breast cancer and demonstrated that epigenetic silencing is a major regulator of CREB3L1 expression loss.[26] In the present study, a significant association was observed between CREB3L1 expression and lung metastases. Jing et al. reported that higher levels of CREB3L1 protein are correlated with increased doxorubicin sensitivity of xenograft RCC tumors in vitro.[27] Upon doxorubicin treatment, CREB3L1 is cleaved so that the $\mathrm{N}$-terminal domain of the protein can reach the nucleus, where it activates the transcription of genes that inhibit cell proliferation[26]. As the first-line chemotherapeutic regimens to treat advanced soft-tissue sarcoma predominantly contain doxorubicin, CREB3L1 has good potential as a useful biomarker of sensitivity to doxorubicin-based palliative chemotherapy for advanced soft-tissue sarcoma.
Effort has been made to identify biomarkers in advanced soft-tissue sarcoma that can predict disease progression and clinical outcomes. Merimsky et al. reported that erbB-4 expression may predict survival in patients with soft-tissue sarcoma treated with chemotherapy.[28] Moreover, Nakatani et al. reported that miR-34a expression may directly influence cell chemo-sensitivity and malignancy in soft-tissue sarcoma.[29] Marklein et al. reported that PI3K inhibition enhances doxorubicin-induced apoptosis in sarcoma cells.[30] However, few markers have been confirmed to predict survival in soft-tissue sarcoma patients.

The results of the present study indicate that CREB3L1 can help identify patients with advanced soft-tissue sarcoma that will respond to doxorubicinbased palliative chemotherapy, especially for those treated with the MAID (AI) regimen. Therefore, advanced soft-tissue sarcoma patients with high CREB3L1 expression may benefit from doxorubicin-based chemotherapy, while those with low CREB3L1 expression may fare better with other chemotherapeutic regimens, radiotherapy or combined treatment. Prospective studies applying this selective approach are needed in the future.

One limitation of the present study is that we did not show a direct role of CREB3L1 in advanced soft-tissue sarcoma. However, our CREB3L1 staining results of clinical samples suggest that an abundance of CREB3L1 is associated with responses to doxorubicin-based palliative chemotherapy. Other limitations of this study include a small sample size for analysis and a retrospective study design. More multi-center prospective researches with independent cohorts for validation are needed to address these limitations.

\section{Conclusions}

The present study suggests that the CAV/IE alternating regimen may be associated with more favorable survival than the MAID (AI) regimen. Our study also indicates that CREB3L1 could be a useful biomarker for predicting the efficacy of doxorubicin-based palliative chemotherapy in advanced soft-tissue sarcoma.

\section{Acknowledgments}

This work was supported by the National Scientific Foundation of China (No. 81572403, 81772863).

\section{Competing Interests}

The authors have declared that no competing interest exists. 


\section{References}

1. Rimondi E, Benassi MS, Bazzocchi A, Balladelli A, Facchini G, Rossi G, et al. Translational research in diagnosis and management of soft tissue tumours. Cancer Imaging. 2016; 16: 13.

2. von Mehren M, Randall RL, Benjamin RS, Boles S, Bui MM, Conrad EU, 3rd, et al. Soft Tissue Sarcoma, Version 2.2016, NCCN Clinical Practice Guidelines in Oncology. Journal of the National Comprehensive Cancer Network : JNCCN. 2016; 14: 758-86

3. Elias A, Ryan L, Sulkes A, Collins J, Aisner J, Antman KH. Response to mesna, doxorubicin, ifosfamide, and dacarbazine in 108 patients with metastatic or unresectable sarcoma and no prior chemotherapy. J Clin Oncol. 1989; 7: 1208-16.

4. Trojani M, Contesso G, Coindre JM, Rouesse J, Bui NB, de Mascarel A, et al. Soft-tissue sarcomas of adults; study of pathological prognostic variables and definition of a histopathological grading system. Int J Cancer. 1984; 33: 37-42.

5. Arndt CA, Nascimento AG, Schroeder G, Schomberg PJ, Neglia JP, Sencer SF, et al. Treatment of intermediate risk rhabdomyosarcoma and undifferentiated sarcoma with alternating cycles of vincristine/doxorubicin/ cyclophosphamide and etoposide/ifosfamide. European journal of cancer (Oxford, England : 1990). 1998; 34: 1224-9.

6. Arndt CA, Hawkins DS, Meyer WH, Sencer SF, Neglia JP, Anderson JR. Comparison of results of a pilot study of alternating vincristine/doxorubicin/cyclophosphamide and etoposide/ifosfamide with IRS-IV in intermediate risk rhabdomyosarcoma: a report from the Children's Oncology Group. Pediatr Blood Cancer. 2008; 50: 33-6.

7. Santoro A, Tursz T, Mouridsen H, Verweij J, Steward W, Somers R, et al. Doxorubicin versus CYVADIC versus doxorubicin plus ifosfamide in first-line treatment of advanced soft tissue sarcomas: a randomized study of the European Organization for Research and Treatment of Cancer Soft Tissue and Bone Sarcoma Group. J Clin Oncol. 1995; 13: 1537-45.

8. Chibon F, Lagarde P, Salas S, Perot G, Brouste V, Tirode F, et al. Validated prediction of clinical outcome in sarcomas and multiple types of cancer on the basis of a gene expression signature related to genome complexity. Nat Med. 2010; 16: 781-7.

9. Coindre JM, Terrier P, Guillou L, Le Doussal V, Collin F, Ranchere D, et al. Predictive value of grade for metastasis development in the main histologic types of adult soft tissue sarcomas: a study of 1240 patients from the French Federation of Cancer Centers Sarcoma Group. Cancer. 2001; 91: 1914-26.

10. Coindre JM, Terrier P, Bui NB, Bonichon F, Collin F, Le Doussal V, et al. Prognostic factors in adult patients with locally controlled soft tissue sarcoma. A study of 546 patients from the French Federation of Cancer Centers Sarcoma Group. J Clin Oncol. 1996; 14: 869-77.

11. Teixeira LE, Araujo ID, de Andrade MA, Gomes RA, Salles PG, Ghedini DF. [Local recurrence in soft tissue sarcoma: prognostic factors]. Rev Col Bras Cir. 2009; 36: 377-81.

12. Murakami T, Kondo S, Ogata M, Kanemoto S, Saito A, Wanaka A, et al. Cleavage of the membrane-bound transcription factor OASIS in response to endoplasmic reticulum stress. J Neurochem. 2006; 96: 1090-100.

13. Denard B, Seemann I, Chen O, Gay A, Huang H, Chen $Y$, et al. The membrane-bound transcription factor CREB3L1 is activated in response to virus infection to inhibit proliferation of virus-infected cells. Cell Host Microbe. 2011; 10: 65-74.

14. Denard B, Lee C, Ye J. Doxorubicin blocks proliferation of cancer cells through proteolytic activation of CREB3L1. Elife. 2012; 1: e00090.

15. Mouridsen HT, Bastholt L, Somers R, Santoro A, Bramwell V, Mulder JH, et al. Adriamycin versus epirubicin in advanced soft tissue sarcomas. A randomized phase II/phase III study of the EORTC Soft Tissue and Bone Sarcoma Group. Eur J Cancer Clin Oncol. 1987; 23: 1477-83.

16. Cormier JN, Pollock RE. Soft tissue sarcomas. CA Cancer J Clin. 2004; 54: 94-109.

17. Kotilingam D, Lev DC, Lazar AJ, Pollock RE. Staging soft tissue sarcoma: evolution and change. CA Cancer J Clin. 2006; 56: 282-91; quiz 314-5.

18. Ogilvie CM, Crawford EA, Slotcavage RL, King JJ, Lackman RD, Hartner L, et al. Treatment of adult rhabdomyosarcoma. Am J Clin Oncol. 2010; 33: 128-31.

19. Maruyama D, Watanabe T, Maeshima AM, Nomoto J, Taniguchi H, Azuma T, et al. Modified cyclophosphamide, vincristine, doxorubicin, and methotrexate (CODOX-M)/ifosfamide, etoposide, and cytarabine (IVAC) therapy with or without rituximab in Japanese adult patients with Burkitt lymphoma (BL) and B cell lymphoma, unclassifiable, with features intermediate between diffuse large B cell lymphoma and BL. Int J Hematol. 2010; 92: 732-43.

20. Elisson LO, Ekberg L. Ifosfamide, doxorubicin, vincristine, and etoposide in small cell lung cancer. Seminars in oncology. 1995; 22: 15-7.

21. Womer RB, West DC, Krailo MD, Dickman PS, Pawel BR, Grier HE, et al. Randomized controlled trial of interval-compressed chemotherapy for the treatment of localized Ewing sarcoma: a report from the Children's Oncology Group. J Clin Oncol. 2012; 30: 4148-54.

22. Grier HE, Krailo MD, Tarbell NJ, Link MP, Fryer CJ, Pritchard DJ, et al, Addition of ifosfamide and etoposide to standard chemotherapy for Ewing's sarcoma and primitive neuroectodermal tumor of bone. N Engl J Med. 2003; 348: 694-701.

23. Sheng JY, Movva S. Systemic Therapy for Advanced Soft Tissue Sarcoma. Surg Clin North Am. 2016; 96: 1141-56.

24. Karavasilis V, Seddon BM, Ashley S, Al-Muderis O, Fisher C, Judson I. Significant clinical benefit of first-line palliative chemotherapy in advanced soft-tissue sarcoma: retrospective analysis and identification of prognostic factors in 488 patients. Cancer. 2008; 112: 1585-91.

25. Mellor P, Deibert L, Calvert B, Bonham K, Carlsen SA, Anderson DH. CREB3L1 is a metastasis suppressor that represses expression of genes regulating metastasis, invasion, and angiogenesis. Mol Cell Biol. 2013; 33: 4985-95.

26. Ward AK, Mellor P Smith SE, Kendall S, Just NA, Vizeacoumar FS, et al. Epigenetic silencing of CREB3L1 by DNA methylation is associated with high-grade metastatic breast cancers with poor prognosis and is prevalent in triple negative breast cancers. Breast Cancer Res. 2016; 18: 12.

27. Denard B, Pavia-Jimenez A, Chen W, Williams NS, Naina H, Collins R, et al. Identification of CREB3L1 as a Biomarker Predicting Doxorubicin Treatment Outcome. PLoS One. 2015; 10: e0129233.

28. Merimsky O, Issakov J, Bickels J, Kollender Y, Flusser G, Soyfer V, et al. ErbB-4 expression in limb soft-tissue sarcoma: correlation with the results of neoadjuvant chemotherapy. European journal of cancer (Oxford, England : 1990). 2002; 38: 1335-42.

29. Nakatani F, Ferracin M, Manara MC, Ventura S, Del Monaco V, Ferrari S, et al. miR-34a predicts survival of Ewing's sarcoma patients and directly influences cell chemo-sensitivity and malignancy. J Pathol. 2012; 226: 796-805.

30. Marklein D, Graab U, Naumann I, Yan T, Ridzewski R, Nitzki F, et al. PI3K inhibition enhances doxorubicin-induced apoptosis in sarcoma cells. PLoS One. 2012; 7: e52898 